\title{
Ação Mucolítica da Bromelina: uma revisão integrativa
}

\author{
Mucolytic action of bromelain: an integrative review \\ Acción mucolítica de la bromelina: uma revisión integradora
}

Eduardo Henrique Barros Ferreira ORCID: https://orcid.org/0000-0002-0072-3378 Universidade de San Lorenzo, Paraguai E-mail: eh1405@hotmail.com Karine Costa Melo

ORCID: https://orcid.org/0000-0001-8253-859X

Universidade Federal do Maranhão, Brasil

E-mail: karinemelo09@gmail.com

Chrisllayne Oliveira da Silva

ORCID: https://orcid.org/0000-0002-0844-0268

Universidade Federal do Maranhão, Brasil

E-mail: chris-layne10@hotmail.com

Jesineide Sousa da Silva

ORCID: https://orcid.org/0000-0003-2930-1694

Centro Universitário de Ciências e Tecnologia do Maranhão, Brasil

E-mail: jesineides@gmail.com

Laila da Silva Mota Vilanova

ORCID: https://orcid.org/0000-0001-8873-5914

Centro Universitário de Ciências e Tecnologia do Maranhão, Brasil

E-mail: lailajoseneto@gmail.com

Francisléia Falcão França Santos Siqueira

ORCID: https://orcid.org/0000-0001-8783-5139

Universidade Estadual do Maranhão, Brasil

E-mail: gpmsaude@gmail.com

Solygardia Albuquerque Maciel Teixeira

ORCID: https://orcid.org/0000-0003-0214-0982

Centro Universitário Nossa Senhora do Patrocínio, Brasil

E-mail: solygardia@bol.com.br

Hálmisson D'Árley Santos Siqueira

ORCID: https://orcid.org/0000-0001-9831-5892

Centro Universitário de Ciências e Tecnologia do Maranhão, Brasil

E-mail: halmisson@yahoo.com.br

Raimundo Nonato Cardoso Miranda Junior

ORCID: https://orcid.org/0000-0003-2937-6143

Centro Universitário de Ciências e Tecnologia do Maranhão, Brasil

E-mail:jrfarmaceutico@hotmail.com

Hádila Giovanna Santos Siqueira Cunha

ORCID: https://orcid.org/0000-0002-8401-6119

Universidade Federal do Maranhão, Brasil

E-mail: hadilagiovanna@ hotmail.com

Jairina Nunes Chaves

ORCID: https://orcid.org/0000-0002-3547-6901

Universidade Estadual do Maranhão, Brasil

E-mail: inanunes@hotmail.com

Rodolfo Ritchelle Lima dos Santos

ORCID: https://orcid.org/0000-0003-0097-6030

Universidade Federal do Piauí, Brasil

E-mail: rodolforitchelle@gmail.com

Camilla Lohanny Azevedo Viana

ORCID: https://orcid.org/0000-0002-4529-3607

Centro Universitário de Ciências e Tecnologia do Maranhão, Brasil Email: camillalohanny@hotmail.com

Marconny Lira da Silva

ORCID: https://orcid.org/0000-0003-0334-9294

Centro Tecnológico de Ensino Múltiplo, Brasil

E-mail: marconnylirads@hotmail.com

Ney Rômulo de Oliveira Paula

ORCID: https://orcid.org/0000-0002-0484-3748

Universidade Federal do Piaú, Brasil

E-mail: neyromulo@ufpi.edu.br 


\begin{abstract}
Resumo
A bromelina possui um potencial anti-inflamatório, antioxidante, propriedades anticâncer e funciona também como um auxiliador no processo de digestão e como um agente cardioprotetor. O objetivo do estudo foi analisar por meio da literatura científica a ação mucolítica da bromelina. Trata-se de uma pesquisa revisão bibliográfica da literatura, na qual foi utilizada para a geração da questão norteadora: "Quais evidências científicas mostram a ação mucolítica da bromelina?". Sete estudos incluídos nesta revisão, todos com abordagem quantitativa dos dados (100\%); concentraram-se principalmente no ano de 2017 (42,9\%) e todos estavam na língua inglesa (100\%), com procedência de estudos publicados na Áustria (42,9\%), o European Journal of Pharmaceutics and Biopharmaceutics concentrou mais publicações, correspondendo a $28,6 \%$. Os estudos abordavam sobre o uso da bromelina associadas com outras substâncias, os efeitos da bromelina, tais como a melhoria da permeabilidade para fármacos, e sua atuação no muco intestinal tornando-o mais permeável. O estudo permitiu expandir os conhecimentos acerca da bromelina ou bromelaína enquanto agente mucolítico, seus potenciais nutracêuticos e ação contra algumas patologias, além de sua possível utilização pela indústria farmacêutica para a criação de drogas que ultrapassem a barreira mucolítica mais facilmente.
\end{abstract}

Palavras-chave: Bromelaína; Muco; Secreções corporais.

\begin{abstract}
Bromelain has anti-inflammatory, antioxidant, anti-cancer properties and also works as an aid in the digestion process and as a cardioprotective agent. The aim of the study was to analyze through the scientific literature the mucolytic action of bromelain. This is a literature review of the literature, which was used to generate the guiding question: "What scientific evidence shows the mucolytic action of bromelain?". Seven studies included in this review, all with a quantitative approach to data (100\%); concentrated mainly in the year $2017(42.9 \%)$ and all were in the English language (100\%), with origin of studies published in Austria (42.9\%), the European Journal of Pharmaceutics and Biopharmaceutics concentrated more publications, corresponding to $28.6 \%$. The studies addressed the use of bromelain associated with other substances, the effects of bromelain, such as improving permeability for drugs, and its action on the intestinal mucus, making it more permeable. The study allowed for the expansion of knowledge about bromelain or bromelain as a mucolytic agent, its nutraceutical potentials and action against some pathologies, in addition to its possible use by the pharmaceutical industry for the creation of drugs that overcome the mucolytic barrier more easily.
\end{abstract}

Keywords: Bromelains; Mucus; Bodily secretions.

\title{
Resumen
}

La bromelina tiene propiedades antiinflamatorias, antioxidantes y anticancerígenas y también actúa como ayuda en el proceso de digestión y como agente cardioprotector. El objetivo del estudio fue analizar a través de la literatura científica la acción mucolítica de la bromelina. Se trata de una revisión bibliográfica de la literatura, que se utilizó para generar la pregunta orientadora: “¿Qué evidencia científica muestra la acción mucolítica de la bromelina?”. Siete estudios incluidos en esta revisión, todos con un enfoque cuantitativo de los datos (100\%); concentrados principalmente en el año 2017 (42,9\%) y todos fueron en lengua inglesa (100\%), con origen de estudios publicados en Austria (42,9\%), la Revista Europea de Farmacéutica y Biofarmacéutica concentró más publicaciones, correspondiente al $28,6 \%$. Los estudios abordaron el uso de la bromelina asociada a otras sustancias, los efectos de la bromelina, como mejorar la permeabilidad de los fármacos, y su acción sobre el moco intestinal, haciéndolo más permeable. El estudio permitió ampliar el conocimiento sobre la bromelina o bromelina como agente mucolítico, su potencial nutracéutico y acción frente a algunas patologías, además de su posible uso por parte de la industria farmacéutica para la creación de fármacos que superen fácilmente la barrera mucolítica.

Palabras clave: Bromelaínas; Moco; Secreciones corporales.

\section{Introdução}

O muco intestinal, formado principalmente pela glicoproteína mucina, funciona como uma barreira protetiva contra bactérias, inibindo inflamações e infecções, entretanto, ele pode diminuir a eficácia da administração de medicamentos por via oral (Hansson, 2012). Estudos apontam que a bromelina, por ser uma enzima proteolítica, possui ação mucolítica, diminuindo a viscosidade do muco (Peixoto et al., 2016).

A bromelina pode ser extraída do abacaxi, uma fruta tropical cultivada de forma abundante na América do Sul, podendo ser consumida in natura ou processada, sendo considerada uma das frutas com imensa utilidade para a fabricação de produtos de grande valia, como antioxidantes, ácidos orgânicos, compostos fenólicos, entre outros (Ali, Hashim, \& Lasekan 2020). 
Podendo ser obtida até das partes não comestíveis do abacaxi, a bromelina possui ainda potencial anti-inflamatório, antioxidante, propriedades anticâncer e funciona também como um auxiliador no processo de digestão e como um agente cardioprotetor (Nor, Ramchandran, Duke \& Vasiljevic, 2016; Zdrojewicz, Chorbińska, Bieżyński, \& Krajewski, 2020). No contexto da pandemia, a bromelina se destacou ainda, por apresentar potencial para atuar no tratamento de Covid-19 (Soares et al., 2021).

Tendo em vista que a bromelina tem potencial para ultrapassar a barreira de muco e aumentar a biodisponibilidade das drogas via oral é de suma importância buscar meios de utiliza-la como uma forma de garantir maior eficácia e segurança. Dessa forma, o presente estudo tem como objetivo analisar por meio da literatura científica a ação mucolítica da bromelina; relatar sobre a ação mucolítica da bromelina na biodisponibilidade farmacológica; e descrever a ação mucolítica da bromelina em pesquisas in vitro e in vivo.

\section{Metodologia}

O presente estudo trata-se de uma pesquisa de revisão bibliográfica da literatura do tipo integrativa, um campo da pesquisa científica que dá suporte a Prática Baseada em Evidências (PBE), que é utilizada amplamente em todas as áreas de saúde, e possui certo rigor metodológico o que lhe atribui maior confiabilidade da síntese de informações sobre um determinado tema (Brasil, 2014).

O tema "Ação mucolítica da bromelina: uma revisão integrativa", determinou a construção da estratégia PICO, que representa um acrônimo para População ou Problema (P), Intervenção (I), Comparação (C) e Desfechos (O-outcomes), na qual foi utilizada para a geração da questão norteadora desta revisão: "Quais evidências científicas mostram a ação mucolítica da bromelina?".

Para a localização dos estudos relevantes, que respondessem à pergunta de pesquisa, utilizou-se de descritores indexados nos idiomas português, inglês e espanhol. Os descritores foram obtidos a partir do Medical Subject Headings (MESH), e dos Descritores em Ciências da Saúde (DeCS).

Consultou-se por meio de descritores e palavras-chave as bases de dados PubMed da National Library of Medicine; BVS (Biblioteca Virtual da Saúde), coordenada pela BIREME e composta de bases de dados bibliográficas produzidas pela Rede BVS, como LILACS, além da base de dados MEDLINE, essas bases foram selecionadas devido ao grande reconhecimento e destaque da sua utilização na área da saúde para busca de evidências científicas.

O elemento $\mathrm{C}$ da estratégia PICO não foi abordado nesta pesquisa, pois esta não tem por objetivo comparar intervenções. Os termos utilizados durante a pesquisa foram classificados e combinados nos bancos de dados resultando em estratégias específicas de cada base. A estratégia de busca eletrônica adotada foi a Booleana, conforme demostrado no Quadro 1.

Quadro 1. Estratégias de busca utilizadas nas bases de dados BIREME e PUBMED.

\begin{tabular}{|c|l|c|}
\hline Base de dados & \multicolumn{1}{|c|}{ Estratégia de busca } & Selecionados \\
\hline $\begin{array}{c}\text { BIREME } \\
\text { (descritores Decs) }\end{array}$ & (Bromelains) AND (Mucus) OR (Bodily Secretions) & 03 \\
\hline $\begin{array}{c}\text { PubMed } \\
(\text { descriptors MeSH) }\end{array}$ & $\begin{array}{l}\text { (Mucus AND ((y_5[Filter]) AND (fft[Filter]))) AND (Bromelains AND ((y_5[Filter]) } \\
\text { AND (fft[Filter])) }\end{array}$ & 04 \\
\hline
\end{tabular}

Fonte: Base de dados (2021).

Como critérios de inclusão utilizaram-se estudos disponíveis em sua totalidade, publicados nos últimos seis anos, de 2016 até 2021, nos idiomas Português, Espanhol e Inglês. Foram excluídos da busca inicial capítulos de livros, resumos, textos 
incompletos, teses, dissertações, monografias, relatos técnicos e outras formas de publicação que não artigos científicos completos.

A análise para seleção dos estudos foi realizada em duas fases, a saber: na primeira, os estudos foram préselecionados segundo os critérios de inclusão e exclusão e de acordo com a estratégia de funcionamento e busca de cada base de dados.

$\mathrm{Na}$ segunda fase os estudos foram analisados quanto ao potencial de participação no estudo, avaliando o atendimento à questão de pesquisa, bem como o tipo de investigação, objetivos, amostra, método, resultados e conclusão. Ao final sete (07) artigos atenderam a questão norteadora e foram adicionados ao estudo.

$\mathrm{Na}$ análise e interpretação dos resultados foram avaliadas as informações coletadas nos artigos científicos e criadas categorias analíticas que facilitou a ordenação e a sumarização de cada estudo. Essa categorização foi realizada de forma descritiva, indicando os dados mais relevantes para o estudo. A pesquisa levou em consideração os aspectos éticos dos estudos quanto às citações, respeitando a autoria das ideias, os conceitos e as definições presentes nos artigos incluídos nesta revisão.

Optou-se pela análise em forma estatística e de forma de texto, utilizando cálculos matemáticos e inferências, que foram apresentados em quadros e tabelas para facilitar a visualização e compreensão.

\section{Resultados}

Dos sete estudos incluídos nesta revisão, todos apresentaram abordagem quantitativa dos dados (100\%); em que três $(42,9 \%)$ concentraram-se principalmente no ano de 2017; e todos estavam na língua inglesa (100\%). Sobre a procedência houve predomínio de estudos publicados na Áustria, correspondente a três $(42,9 \%)$ trabalhos. No tocante do periódico o European Journal of Pharmaceutics and Biopharmaceutics concentrou mais publicações, correspondendo a 28,6\%; os demais periódicos obtiveram 14,3\% cada, conforme demonstrado na Tabela 1. Os estudos foram avaliados abordavam principalmente sobre enzimas proteolíticas e a ação mucolítica da bromelina na prática clínica, como mostra o Quadro 2. 
Tabela 1. Análise descritiva das produções científicas acerca do uso de bromelina como agente mucolítico. Teresina, PI, Brasil, 2021. $(\mathrm{N}=7)$.

\begin{tabular}{llc}
\hline Variáveis & $\mathbf{N}$ & $\%$ \\
\hline Base de dados & 03 & 42,9 \\
BIREME & 04 & 57,1 \\
PUBMED & & 100,0 \\
Abordagem do estudo & 07 & 28,6 \\
Quantitativo & & 42,9 \\
Ano & 02 & 14,3 \\
2015 & 03 & 14,3 \\
2017 & 01 & 100,0 \\
2018 & 01 & \\
2020 & & \\
Idioma & 07 & 12,9 \\
Inglês & & 14,3 \\
País & 03 & 14,3 \\
Áustria & 01 \\
Reino Unido & 01 & 28,6 \\
Estados Unidos & 01 & \\
Austrália & 02 & 14,3 \\
Periódicos & & 28,6 \\
Enternational Journal of Pharmaceutics & 01 & 14,3 \\
Colloids and Surfaces B: Biointerface & 02 & 14,3 \\
Translational Research & 01 & 14,3 \\
International Journal of Medical Sciences & 01 & 14,3 \\
Pleura and Peritoneum & 01 & 01 \\
\hline
\end{tabular}

Fonte: Dados da pesquisa (2021). 
Quadro 2. Publicações incluídas segundo autor/ano, título, objetivo geral e principais resultados. Teresina, PI, Brasil, 2021.

$(\mathrm{N}=7)$.

\begin{tabular}{|c|c|c|c|}
\hline AUTOR/ANO & TÍTULO & OBJETIVO GERAL & PRINCIPAIS RESULTADOS \\
\hline $\begin{array}{l}\text { Dilly et al. } \\
(2021)\end{array}$ & $\begin{array}{l}\text { Improved } \\
\text { chemosensitivity } \\
\text { following mucolytic } \\
\text { therapy in patient-derived } \\
\text { models of mucinous } \\
\text { appendix cancer }\end{array}$ & $\begin{array}{l}\text { Avaliar o papel citoprotetor do muco } \\
\text { extracelular e os efeitos mucolíticos } \\
\text { de BRO + NAC ex vivo usando } \\
\text { explantes tumorais mucinosos } \\
\text { derivados de pacientes. }\end{array}$ & $\begin{array}{l}\text { A combinação de BRO + NAC no muco extracelular em } \\
\text { modelos clinicamente relevantes de MCP foi eficiente. A } \\
\text { conversão de tumores mucinosos sólidos em ascite mucinosa, } \\
\text { diminui o volume do tumor e permite a drenagem } \\
\text { minimamente invasiva de tumores liquidificados. A lise do } \\
\text { muco extracelular remove o revestimento mucinoso protetor } \\
\text { que envolve as células cancerosas e melhora a entrega / } \\
\text { eficácia do fármaco quimioterápico nas células cancerosas. }\end{array}$ \\
\hline $\begin{array}{l}\text { Efiana, Phan, } \\
\text { Wicaksono, \& } \\
\text { Bernkop- } \\
\text { Schnürch, } \\
\text { (2018) }\end{array}$ & $\begin{array}{l}\text { Mucus permeating self- } \\
\text { emulsifying drug delivery } \\
\text { systems (SEDDS): About } \\
\text { the impact of mucolytic } \\
\text { enzymes }\end{array}$ & $\begin{array}{l}\text { Melhorar as propriedades de } \\
\text { permeação de muco de sistemas de } \\
\text { liberação de fármacos } \\
\text { autoemulsionantes (SEDDS) por meio } \\
\text { da ancoragem de bromelaína } \\
\text { lipidizada, papaína e tripsina usando } \\
\text { cloreto de palmitoila. }\end{array}$ & $\begin{array}{l}\text { A maior porcentagem de permeação foi alcançada pela } \\
\text { introdução de } 5 \% \text { de papaína-palmitato em SEDDS. Pode } \\
\text { aumentar a permeação de muco de SEDDS em muco intestinal } \\
\text { suíno } 4,6 \text { vezes e } 2 \text { vezes, conforme avaliado por difusão } \\
\text { Transwell e rotação método do tubo, respectivamente. Conclui- } \\
\text { se que a permeação do muco de SEDDS pode ser fortemente } \\
\text { melhorada por incorporação de conjugados de enzima- } \\
\text { palmitato. }\end{array}$ \\
\hline $\begin{array}{l}\text { Mahmood, } \\
\text { Laffleur, } \\
\text { Leonaviciute, \& } \\
\text { Bernkop- } \\
\text { Schnürch, } \\
\text { (2017) }\end{array}$ & $\begin{array}{l}\text { Protease-functionalized } \\
\text { mucus penetrating } \\
\text { microparticles: } \text { In-vivo } \\
\text { evidence for their } \\
\text { potential }\end{array}$ & $\begin{array}{l}\text { Explorar se a imobilização de } \\
\text { proteases em micropartículas poderia } \\
\text { resultar em sua maior penetração no } \\
\text { muco. }\end{array}$ & $\begin{array}{l}\text { Estudos de penetração do muco mostraram uma permeação } \\
4,27 \text { e } 2,21 \text { vezes maior de PAA-PAP carregado com FDA e } \\
\text { micropartículas de PAA-BROM em comparação com as } \\
\text { micropartículas de PAA, respectivamente. Uma análise in-vitro } \\
\text { na mucosa intestinal suína descrita até } 16 \text { e Grau de retenção } \\
7,35 \text { vezes maior e, além disso, durante a avaliação in vivo em } \\
\text { ratos Sprague-Dawley um } 3,35 \text { e comportamento de penetração } \\
2,07 \text { vezes maior foi observado no intestino delgado para PAA- } \\
\text { PAP e PAA-BROM micropartículas em comparação com } \\
\text { micropartículas de PAA, respectivamente. }\end{array}$ \\
\hline $\begin{array}{c}\text { Pillai, Akhter \& } \\
\text { Morris (2017) }\end{array}$ & $\begin{array}{l}\text { Assessment of a novel } \\
\text { mucolytic solution for } \\
\text { dissolving mucus in } \\
\text { pseudomyxoma peritonei: } \\
\text { an ex vivo and in vitro } \\
\text { study. }\end{array}$ & $\begin{array}{l}\text { Comparar o efeito mucolítico de duas } \\
\text { fórmulasções (controle: bromelaína } \\
300 \mu \mathrm{g} / \mathrm{mL}+\mathrm{N} \text {-acetilcisteína } \\
250 \mathrm{mM} \text {; teste: bromelaína } 200 \mu \mathrm{g} / \\
\mathrm{mL}+\text { cisteamina } 200 \mathrm{mM} \text { ) }\end{array}$ & $\begin{array}{l}\text { A solubilização foi mais rápida com o teste vs. formulação de } \\
\text { controle ( } 90 \text { vs. } 180 \text { min) para dissolver os tampões de mucina } \\
\text { mole (p <0,05). A solução de teste também foi mais eficaz na } \\
\text { dissolução dos tampões de muco duro pareado ao controle } \\
(82,5 \pm 2,74 \% \text { vs. } 36,33 \pm 3,27 \%) \text {. Tudo } \\
\text { tipos de mucina desintegrados em lavagem peritoneal } \\
\text { simulada. A citotoxicidade da solução de teste na linha celular } \\
\text { HT29 foi dependente do tempo. }\end{array}$ \\
\hline $\begin{array}{l}\text { Pillai, Akhter, } \\
\text { Mekkawy, Chua, } \\
\text { \& Morris, } \\
\text { (2017) }\end{array}$ & $\begin{array}{lr}\text { Physical and } & \text { chemical } \\
\text { characteristics of mucin } \\
\text { secreted } & \text { by } \\
\text { pseudomyxoma } & \text { peritonei } \\
\text { (PMP) } & \end{array}$ & $\begin{array}{l}\text { Investigar as características físicas e } \\
\text { químicas da intermucina, a fim de } \\
\text { reformular um mucolítico eficaz para } \\
\text { todas as mucinas. }\end{array}$ & $\begin{array}{l}\text { Tanto a turbidez quanto a viscosidade cinemática e o teor de } \\
\text { ácido siálico aumentaram linearmente conforme a dureza da } \\
\text { mucina aumentou. No entanto, densidade, hidratação, proteína, } \\
\text { glicose, lipídio e sulfidrila e o conteúdo de dissulfeto diminuiu } \\
\text { linearmente com o aumento da dureza da mucina. A diferença } \\
\text { na textura e dureza da mucina pode ser devido ao conteúdo } \\
\text { celular, hidratação, distribuição de glicose, proteína, lipídios, } \\
\text { tiol e MUC. Mucina mole é feita exclusivamente de } \\
\text { glicoproteína enquanto as outras continham materiais celulares. }\end{array}$ \\
\hline $\begin{array}{l}\text { Sousa et al. } \\
(2015)\end{array}$ & $\begin{array}{l}\text { Nanoparticles decorated } \\
\text { with proteolytic enzymes, } \\
\text { a promising strategy to } \\
\text { overcome the mucus } \\
\text { barrier }\end{array}$ & $\begin{array}{l}\text { Comparar as características das } \\
\text { nanopartículas (NPs) conjugadas com } \\
\text { papaína (PAP) com bromelaína } \\
(\mathrm{BRO}) .\end{array}$ & $\begin{array}{l}\text { Em todos os testes BRO modificados NPs exibem o efeito mais } \\
\text { significativo na alteração e na estrutura do muco e o melhor } \\
\text { desempenho na permeação em uma camada de muco fresco em } \\
\text { comparação com NPs conjugados com PAP. A modificação } \\
\text { com BRO pode melhorar ainda mais a permeabilidade de PAA } \\
\text { NPs e, portanto, este sistema pode ser explorado como drogas } \\
\text { capazes de transportar a carga terapêutica através do muco, } \\
\text { barreira e, consequentemente, capaz de melhorar a } \\
\text { biodisponibilidade de medicamentos administrados por via } \\
\text { oral. }\end{array}$ \\
\hline $\begin{array}{l}\text { Wilcox, Rooij, } \\
\text { Chater, Souza, } \\
\text { \& Pearson } \\
\text { (2015) }\end{array}$ & $\begin{array}{l}\text { The effect of nanoparticle } \\
\text { permeation on the bulk } \\
\text { rheological properties of } \\
\text { mucus from the small } \\
\text { intestine. }\end{array}$ & $\begin{array}{l}\text { Investigar os efeitos de dois nano- } \\
\text { sistemas de partículas, um poli (ácido } \\
\text { lático-co-glicólico) (PLGA) e uma } \\
\text { nanopartícula de poli (ácido acrílico), } \\
\text { (PAA) decorada com bromelaína } \\
\text { (BRO), nas propriedades reológicas } \\
\text { em massa de suínos no muco } \\
\text { intestinal do intestino delgado. }\end{array}$ & $\begin{array}{l}\text { Tratamento com nanopartículas diminuí a força do gel de muco } \\
\text { com PAA-BRO reduzindo ao máximo. Nanopartículas de } \\
\text { PAA-BRO resultaram em liberação de glicoproteína } \\
\text { aumentada da rede de gel, enquanto o muco permaneceu um } \\
\text { gel e exibiu um estresse de decomposição semelhante para } \\
\text { controlar o muco. Portanto, seria possível usar a bromelaína } \\
\text { para aumentar a permeabilidade das nanopartículas através do } \\
\text { muco, sem destruir o gel e deixando o substrato } \\
\text { mucosa desprotegida. }\end{array}$ \\
\hline
\end{tabular}

Fonte: Dados da pesquisa (2021).

Os estudos abordavam sobre o uso da bromelina associadas com outras substâncias, os efeitos da bromelina, tais como a melhoria da permeabilidade para fármacos, e sua atuação no muco intestinal tornando-o mais permeável, também ressaltaram 
sobre nanopartículas e bromelina e como a bromelina pode atuar eliminando tumores, algo que ainda está em estudo, mais que se demonstrasse eficácia seria promissor na prática clínica.

\section{Discussão}

\subsection{Ação mucolítica da bromelina}

A bromelina em forma de nanopartículas é capaz de ampliar a permeabilidade da barreira de muco, degradando-a, sem causar a perda de sua função vital protetora. O rompimento da barreira acontece por meio do deslocamento de componentes do muco, quebrando algumas ligações de hidrogênio (Wilcox et al., 2015). Ao clivar a estrutura da mucosa, essas partículas reduzem a viscosidade do muco e abrem caminho para a passagem das drogas (Mahmood et al., 2017).

Em seu trabalho, Sousa et al. (2015), compararam características de nanopartículas formadas por papaína e nanopartículas formadas por bromelina no rompimento do muco, demonstrando que a bromelina apresentou uma permeação maior, o que foi associado à sua maior estabilidade depois da conjugação do polímero, fazendo com que ela perca menor atividade enzimática. Ao investigar o efeito de nanopartículas de poli (ácido acrílico) decoradas com bromelina na reologia do muco, Wilcox et al. (2015) encontraram resultado semelhante, no qual as nanopartículas minimizaram a força do gel do muco e reduziram a viscosidade do mesmo.

Além da carga superficial (potencial zeta), preferencialmente negativo, o que é necessário para que elas penetrem no muco de forma direta, o tamanho das partículas é fundamental para que elas consigam romper o muco, no qual, as partículas com dimensão de <200 nm são mais favoráveis para alcançar esse objetivo (Efiana et al., 2018; MAHMOOD et al., 2017), características que fazem com que as nanopartículas de bromelina apresentem ação mucolítica.

\subsection{Ação mucolítica na biodisponibilidade de fármacos}

Dentre todas as vias de administração conhecidas e utilizadas na prática clínica, a oral é a mais favorável, no entanto, a baixa biodisponibilidade oral de vários medicamentos, leva-se a criação de estratégias capazes de melhorar a solubilidade do medicamento e absorção dos mesmos. Uma das principais causas de baixo consumo de drogas orais e da biodisponibilidade é a presença de uma barreira que cobre toda a superfície face do trato gastrointestinal, que é a barreira de muco. Essa barreira é uma camada de gel, com uma matriz complexa formada principalmente por mucina (Sousa et al. 2015).

$\mathrm{O}$ trajeto de fármacos pela mucosa representa uma estratégia eficaz na absorção de uma variedade de ingredientes farmacêuticos ativos, e desta forma do ponto de vista terapêutico, as partículas que compõe esses fármacos, como micropartículas, possuem vantagens de uma maior carga útil de drogas e com maior eficiência de encapsulamento e possibilidade de liberação dos princípios ativos contidos nas drogas (Mahmood et al. 2017).

Nos estudos de Sousa et al. (2015) com teste utilizando a bromelina em pesquisa in-vivo, obteve resultados através de diversas técnicas que existe uma forte correlação do uso de bromelina para biodisponibilidade farmacológica, pois proporciona um efeito mais significativo na alteração da estrutura do muco, possibilitando um melhor desempenho na permeação e reduzindo a barreira imposta pelo muco, o que possibilita melhor absorção e aproveitamento dos fármacos utilizados.

A utilização de micropartículas de Bromelina e papaína em estudo in-vivo, demonstraram resultados positivos no aumento da viscosidade do muco intestinal, isso permite que as micropartículas de bromelina e papaína consigam ultrapassar as barreiras de muco e alcançar o revestimento de absorção permitindo uma ampla gama de aplicações na administração de drogas por meio da mucosa (Mahmood et al. 2017).

Através de um sistema de entrega de drogas autoemulsionantes (SEDDS), com o uso de enzima-palmitato com bromelina e papaína, foi demonstrado melhora na capacidade de permear o muco intestinal devido à capacidade das enzimas 
de clivar a glicoproteína no muco. Além disso, SEDDS como sistemas transportadores de drogas promissores têm capacidade de aproximar drogas lipofílicas da absorção na membrana (Efiana et al. 2018).

Essa integração de palmitato de bromelaína, palmitato de papaína bem como palmitato de tripsina em SEDDS aumentou significativamente permeação de muco, e essa descobertas sugerem que os conjugados enzima-palmitato (SEDDS) podem ser usados como sistemas de transportadores de penetração de muco para desenvolvimento posterior de entrega de drogas e absorção mais eficiente (Efiana et al. 2018).

Uma descoberta interessante foi apresentada nos estudos de Dilly et al. (2021) na qual apontaram que a terapia mucolítica aumenta a administração do medicamento às células cancerosas e melhora a quimiossensibilidade. A Mucólise antes da exposição ao medicamento quimioterápico nos experimentos ex vivo e in vitro permitiram melhorias administração de drogas.

Além de propriedades mucolíticas, a bromelina foi anteriormente mostrada com efeitos citotóxicos diretos contra linhagens de células cancerígenas, e reduziu com sucesso o crescimento do tumor mucinoso no modelo PDX de ratos de carcinoma mucinoso apendicular peritoneal (MCP) inicial. Essa estratégia de tratamento pode ter um papel clínico como terapia adjuvante para pacientes MCP ressecados. A bromelina pode prevenir o crescimento de tumor mucinosos por via mucolítica e ser anticâncer, porém a bromelina tem propriedades antiplaquetárias e trombolíticas que podem levar a consequências adversas da terapia prolongada, por isso são necessários mais estudos, voltados para teste de eficiência e dosagem adequada que permitam essa funcionalidade (Dilly et al. 2021).

\subsection{Ação mucolítica da bromelina e associações em pesquisas in vitro e in vivo}

A bromelina tem sido muito utilizada pela indústria alimentícia e farmacológica por apresentar grandes benefícios para a saúde de quem a consome. Neste aspecto, a bromelina enquanto agente mucolítico tem sido testada em pesquisas in vitro e in vivo, a fim de avaliar seus efeitos e determinar a melhor forma de utilizar a substância (Pillai, Akhater, \& Morris, 2017).

Em uma pesquisa in vivo, realizada utilizando ratos e intestinos suínos, Mahmood et al. (2017) conduziram os experimentos repetidas vezes para comprovar que, a bromelina associada papaína apresentaram grande potencial mucolítico onde, foi evidenciado que a combinação não apresentou toxicidade e expôs grande capacidade para aumentar a permeação de substâncias, oferecendo potencial para diversas aplicações na administração de drogas.

Como o efeito das drogas administradas por via oral, muitas vezes, é prejudicado devido a sua absorção começar na boca e à ação protetora que o muco intestinal desempenha. Wilcox et al. (2015), em sua pesquisa in vivo, demostrou que a bromelina e a papaína utilizadas concomitantemente aumentaram a permeabilidade sem deixar a mucosa desprotegida.

Já um estudo in vitro realizado com bromelina e cisteamina na muscina demostrou um efeito de citotoxidade quando utilizado em células tumorais de câncer colorretal. Já o estudo experimental in vivo com a bromelina e a cisteamina não apresentou o efeito negativo e alcançou um efeito mucolítico (Pillai, Akhater, \& Morris, 2017). E Dilly et al. (2021) afirmam que a bromelina e acetilcisteína evidenciaram uma atuação efetiva para a diminuição da massa tumoral na região abdominal de camundongos.

\section{Considerações Finais}

Este estudo permitiu avaliar as evidências científicas acerca da ação mucolítica da enzima proteolítica existente no abacaxi, a bromelina. Onde foram analisados, por meio da literatura científica, os benefícios da bromelina enquanto agente mucolítico, sua associação com outras enzimas e ainda, sua influência positiva sobre a biodisponibilidade farmacológica de drogas administradas por via oral. 
Diante das evidências encontradas, torna-se claro o potencial da bromelina tanto como nutracêutico quanto para utilização em fármacos, já que a substância pode aumentar a permeabilidade do muco intestinal sem prejudicar o organismo. Foi observado ainda que, a bromelina juntamente com outras substâncias tem potencial para reduzir massas tumorais.

Como limitações da pesquisa tem-se o fato de não existiram na literatura, muitos estudos sobre a temática, outra dificuldade que pode ser citada é a escassez de estudos realizados com humanos, sendo a maior parte das pesquisas realizadas in vitro ou com pequenos animais. Além disso, por se tratar de se tratar de um tema que não é frequentemente testado em seres humanos, alguns resultados de testes em humanos podem divergir da literatura.

Por fim, o estudo permitiu expandir os conhecimentos acerca da bromelina ou bromelaína enquanto agente mucolítico, seus potenciais nutracêuticos e ação contra algumas patologias, além de sua possível utilização pela indústria farmacêutica para a criação de drogas que ultrapassem a barreira mucolítica mais facilmente. Dessa forma, considera-se que mais pesquisas devem ser realizadas nesta linha de investigação a fim de provar a importância da bromelina para a saúde e suas diversas formas de utilização.

\section{Referências}

Ali, M. M., Hashim, N., Aziz, S. A., \& Lasekan, O. (2020). Pineapple (Ananas comosus): A comprehensive review of nutritional values, volatile compounds, health benefits, and potential food products. Food Research International,137, 1-13.

Brasil. Ministério da Saúde. Diretrizes metodológicas: elaboração de diretrizes clínicas. Ministério da Saúde, 2014.

Dilly, A. K., Honick, B. D., Frederick, R., Elapavaluru, A., Velankar, S., Makala, H., \& Choudry, H. A. (2021). Improved chemosensitivity following mucolytic therapy in patient-derived models of mucinous appendix cancer. Translational research: the journal of laboratory and clinical medicine, $229,100-$ 114.

Efiana, N. A., Phan, T. N. Q., Wicaksono, A. J., \& Bernkop-Schnürch, A. (2018). Mucus permeating self-emulsifying drug delivery systems (SEDDS): About the impact of mucolytic enzymes. Colloids and surfaces. B, Biointerfaces, 161, 228-235.

Hansson, G. C. (2012) Role of mucus layers in gut infection and inflammation. Curr Opin Microbiol, 15(1), 7-62.

Mahmood, A., Laffleur, F., Leonaviciute, G., \& Bernkop-Schnürch, A. (2017). Protease-functionalized mucus penetrating microparticles: In-vivo evidence for their potential. International journal of pharmaceutics., 532(1), 177-184.

Nor, M. Z. M., Ramchandran, L., Duke, M., \& Vasiljevic, T. (2016). Separation of bromelain from crude pineapple waste mixture by a twostage ceramic ultrafiltration process. Food and Bioproducts Processing, 98, 1-38.

Peixoto, D. M., Rizzo, J. A., Schor, D., Silva, A. R., Oliveira, D. C. D., Solé, D., \& Sarinho, E. (2016). Uso do mel de abelha associado ao Ananas comosus (Bromelin) no tratamento da tosse irritativa aguda. Rev. paul. pediatr., 34(4), 412-417.

Pillai, K., Akhter, J., \& Morris, D. L. (2017). Assessment of a novel mucolytic solution for dissolving mucus in pseudomyxoma peritonei: an ex vivo and in vitro study. Pleura and peritoneum., 2(2), 111-117.

Pillai, K., Akhter, J., Mekkawy, A., Chua, T. C., \& Morris, D. L. (2017). Physical and chemical characteristics of mucin secreted by pseudomyxoma peritonei (PMP). International journal of medical sciences., 14(1), 18-28.

Soares, A. N., Melo, K. C., Da Silva, W. C., Da Silva, C. O., Da Silva, R. A., Da Silva, K. G. S., \& Souza, F. D. C. A. (2021). Potenciais efeitos imunomoduladores de nutracêuticos no tratamento de Covid-19. International Journal of Development Research, 11(4), 46233-46238.

Sousa, I. P., Cattoz, B., Wilcox, M. D., Griffiths, P. C., Dalgliesh, R., Rogers, S., \& Bernkop-Schnürch, A. (2015). Nanoparticles decorated with proteolytic enzymes, a promising strategy to overcome the mucus barrier. European journal of pharmaceutics and biopharmaceutics., 97, $257-264$.

Wilcox, M. D., Rooij, L. K. V., Chater, P. I., Souza, I. P. D., \& Pearson, J. P. (2015). The effect of nanoparticle permeation on the bulk rheological properties of mucus from the small intestine. European journal of pharmaceutics and biopharmaceutics., 96, 484-487.

Zdrojewicz, Z., Chorbińska, J., Bieżyński, B., \& Krajewski, P. (2018). Health-promoting properties of pineapple. Pediatr Med Rodz, 14(2),133-142. 